\title{
In vitro stress effect on degradation and drug release behaviors of basic fibroblast growth factor - poly(lactic-co-glycolic-acid) microsphere
}

This article was published in the following Dove Press journal:

Drug Design, Development and Therapy

25 January 2016

Number of times this article has been viewed

Yan Xiong'
Zeping Yu'
Yun Lang'
Juanyu Hu'
Hong Li'
Yonggang Yan'
Chongqi Tu'
Tianfu Yang'
Yueming Song'
Hong Duan'
Fuxing Pei'
'Department of Orthopedics, West
China Hospital, Sichuan University,
Chengdu, Sichuan, People's Republic of
China; 'Laboratory of Biomechanical
Engineering, Sichuan University,
Chengdu, Sichuan, People's Republic
of China

Correspondence: Hong Duan; Fuxing Pei Department of Orthopedics, West China Hospital, Sichuan University, 37 Guo Xue Lane, Chengdu, Sichuan 6I0064, People's Republic of China

Tel +86 288542 2578; +8628 85422573

Email duanhong1970@I26.com;

peifuxingpeifx@163.com
Objective: To study the degradation and basic fibroblast growth factor (bFGF) release activity of bFGF - poly(lactic-co-glycolic-acid) microsphere (bFGF-PLGA MS) under stress in vitro, including the static pressure and shearing force-simulating mechanical environment of the joint cavity.

Method: First, bFGF-PLGA MSs were created. Meanwhile, two self-made experimental instruments (static pressure and shearing force loading instruments) were initially explored to provide stress-simulating mechanical environment of the joint cavity. Then, bFGF-PLGA MSs were loaded into the two instruments respectively, to study microsphere degradation and drug release experiments. In the static pressure loading experiment, normal atmospheric pressure loading (approximately 0.1 MPa), 0.35 MPa, and 4.0 MPa pressure loading and shaking flask oscillation groups were designed to study bFGF-PLGA MS degradation and bFGF release. In the shearing force loading experiment, a pulsating pump was used to give the experimental group an output of $1,000 \mathrm{~mL} / \mathrm{min}$ and the control group an output of $10 \mathrm{~mL} / \mathrm{min}$ to carry out bFGF-PLGA MS degradation and drug release experiments. Changes of bFGF-PLGA MSs, including microsphere morphology, quality, weight-average molecular weight of polymer, and microsphere degradation and bFGF release, were analyzed respectively.

Results: In the static pressure loading experiment, bFGF-PLGA MSs at different pressure were stable initially. The trend of molecular weight change, quality loss, and bFGF release was consistent. Meanwhile, microsphere degradation and bFGF release rates in the $4.0 \mathrm{MPa}$ pressure loading group were faster than those in the normal and $0.35 \mathrm{MPa}$ pressure loading groups. It was the fastest in the shaking flask group, showing a statistically significant difference $(P<0.0001)$. In the shearing force loading experiment, there were no distinctive differences in the rates of microsphere degradation and bFGF release between experimental and control group. Meanwhile, microsphere degradation and bFGF release rates by shaking flask oscillation were obviously faster than those by shearing force only $(P<0.0001)$.

Conclusion: There are significant effects on bFGF-PLGA MS degradation and bFGF release due to the interaction between extraction stress and time. Static pressure has a conspicuous influence on bFGF-PLGA MS degradation and release, especially at a pressure of 4.0 MPa. The shearing force has a slight effect on bFGF-PLGA MS degradation and drug release. On the contrary, shaking flask oscillation has a significantly distinctive effect.

Keywords: microsphere, basic fibroblast growth factor, bFGF, poly(lactic-co-glycolic-acid), PLGA, stress, drug release

\section{Introduction}

Basic fibroblast growth factor (bFGF) is a member of the FGF family of proteins, which has been studied in embryonic development, nerve nutrition and regeneration, bone and muscle growth, blood vessel formation, wound healing, and tumor growth 
and differentiation. ${ }^{1-7}$ However, because of its instability at high temperatures and in acidic environments its short half-life of 3-5 minutes in vivo, bFGF cannot meet clinical application criteria satisfactorily. Poly(lactic-co-glycolicacid) (PLGA), which is a biodegradable material, has been widely used in pharmaceutical industry as a carrier to load drugs and lengthen sustained release. Over the past few decades, PLGA microspheres (MSs), formulated into different devices for loading a variety of drugs or reagents such as vaccines, antibiotics, peptides, proteins, and micromolecules, have been used to treat disease and promote tissue regeneration and repair. ${ }^{8-10}$ They can also be used for cartilage injury regeneration and repair, as the cartilage lacks good self-repairing ability. ${ }^{11-15}$

Generally, because of low self-repair capability, articular cartilage injury is a common and severe disease that seriously affects physical function and quality of life. ${ }^{11}$ The current methods of repair include the application of exogenous growth factors, as have been reported in previous studies. ${ }^{12-16}$ The bFGF-PLGA MS could be injected into the joint cavity to promote cartilage defect healing. However, the complex environment of joint cavity, especially joint movement, impacts bFGF-PLGA MS degradation and bFGF release.

It is reported that the main degradation mechanism of PLGA microspheres, injected into joint cavity to repair articular cartilage injury, is hydrolytic decomposition, which in turn influenced by the partial microenvironment such as temperature, $\mathrm{pH}$, and enzymes. ${ }^{17-19}$ In addition, it can be affected by stress factors such as static pressure of joint cavity and shearing force. Studies have reported that the highest pressure of hip joint cavity is $0.35 \mathrm{MPa}$ and that of the knee joint cavity is 3.8-4.0 MPa. ${ }^{20-22} \mathrm{In}$ both in vitro and in vivo studies, the stress effects on degradation of biomaterial have been a matter of concern for years. Bos et $\mathrm{a}^{23}$ showed that when biomaterial was applied to weight-bearing parts of the cancellous bone fractures, the loading parts of the material had a faster degradation rate and loss of strength. Korin et $\mathrm{al}^{24}$ revealed that shearing stress caused by an intravascular thrombus could break up the nanoparticles quickly to release thrombolytic drugs for the treatment of obstructive blood vessel diseases. However, there is still lack of research on the effects of stress on PLGA microsphere degradation and drug release in joint cavities. Thus, the purpose of this paper is to evaluate the effects of stress in vitro on bFGF-PLGA MS degradation and drug release, and to provide relevant data for further research.

\section{Materials and methods bFGF-PLGA MS preparation}

The bFGF freeze-dried powder was supplied by the Biopharmaceutical R\&D Center of Jinan University, People's Republic of China. The PLGA, with a 50:50 copolymer ratio and $20 \mathrm{kDa}$ average molecular weight, was prepared by the Department of Organic Chemistry of Chengdu Institute, Chinese Academy of Sciences. The bFGF-loaded microspheres were prepared by solvent extraction/double emulsion (w/o/w) method and conserved by freeze-drying. ${ }^{4}$ The bFGFPLGA MSs prepared by this method were spherical (even and uniform) particles (mean particle size: $5.22 \pm 1.43 \mu \mathrm{m}$ ) that did not adhere to each other (Figure 1). The bFGF loading rate achieved was $(48.10 \pm 1.03) \times 10^{-3} \%(\mathrm{~g} / \mathrm{g})$.

\section{Equipment and experimental processes}

According to previous studies regarding the static pressure of knee and hip joints cavity, ${ }^{20-22}$ self-made equipment, composed of pressure tanks, chemical reaction pressure kettle (Shandong, People's Republic of China), and pressure control valve (Shanghai, People's Republic of China), normal atmospheric pressure loading (approximately 0.1 MPa), 0.35 MPa, and 4.0 MPa pressure loading (Figure 2). The degradation and drug release studies of bFGF-PLGA MSs were performed under those three pressure conditions, or in a shaking flask oscillator (thermostatic water bath oscillator, Jiangsu, People's Republic of China). The detailed experimental process is given in Figure 2: 1) the four group's tubes were marked 1-24a, 1-24b, 1-24c, and 1-24d. Approximately $5 \mathrm{mg}$ bFGF-PLGA MS and $10 \mathrm{~mL}$ phosphate-buffered saline

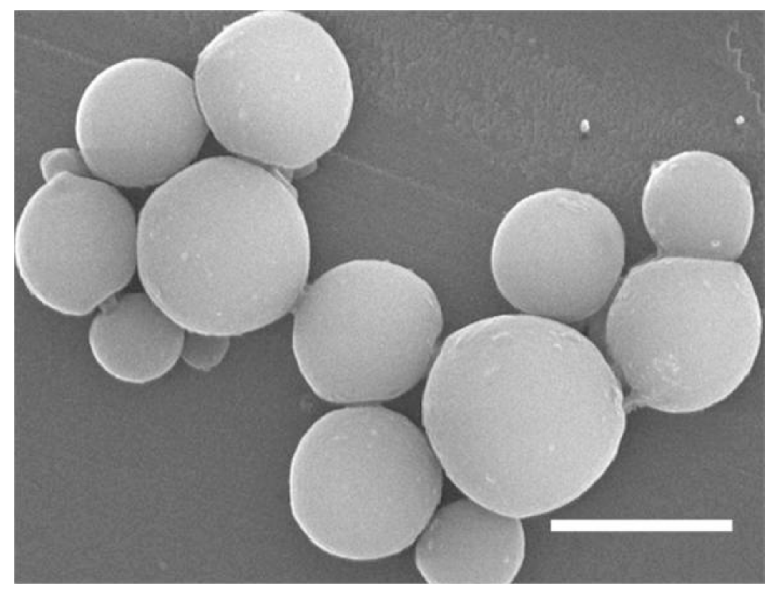

Figure I The morphology of bFGF-PLGA MS (observed by an FEI Quanta 200 scanning electron microscope; FEI, Hillsboro, OR, USA).

Note: Scale bar, $5 \mu \mathrm{m}$.

Abbreviation: bFGF-PLGA MS, basic fibroblast growth factor - poly(lactic-coglycolic-acid) microsphere. 


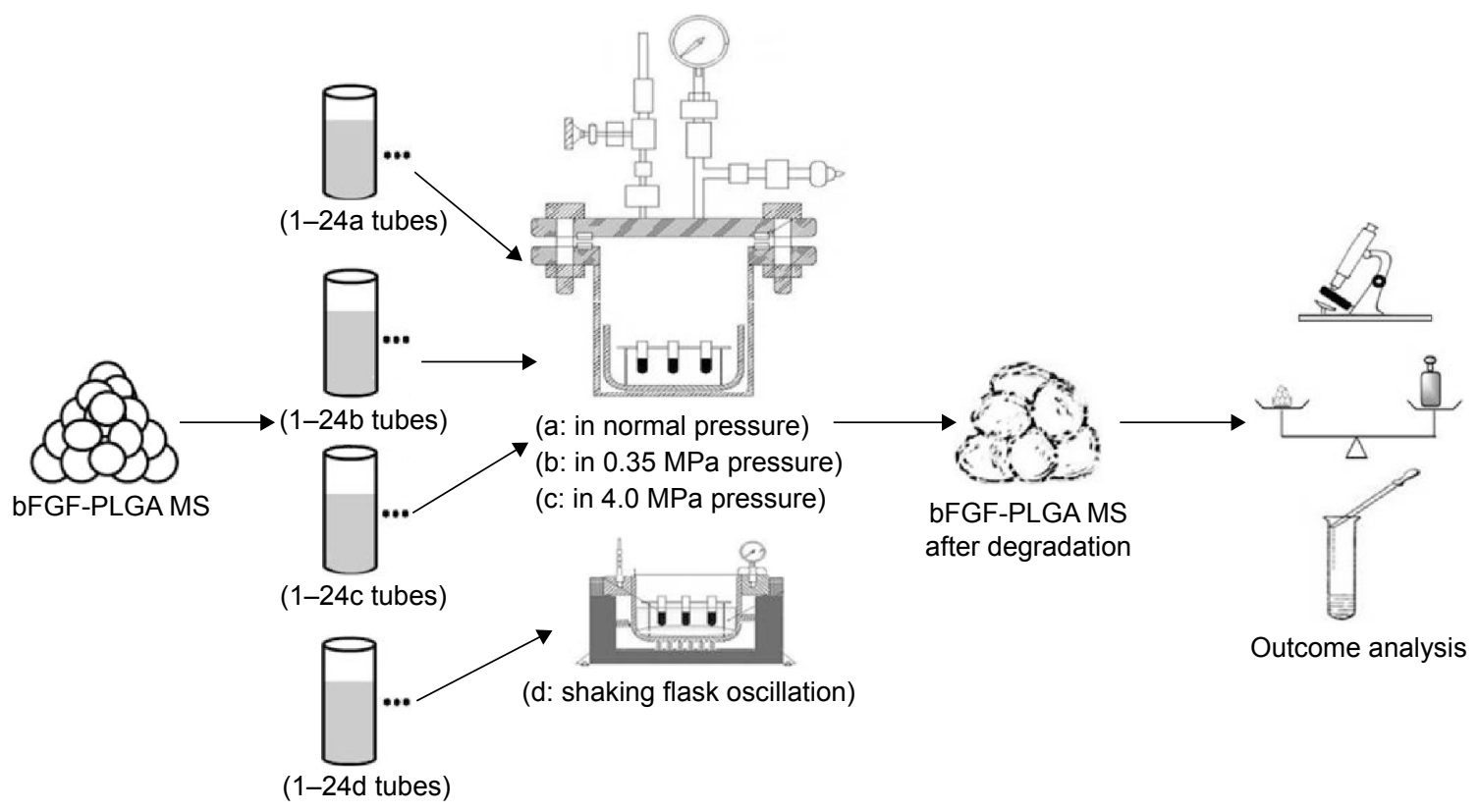

Figure 2 The equipment and experimental process of bFGF-PLGA MS degradation and drug release in static pressure loading experiment. Abbreviation: bFGF-PLGA MS, basic fibroblast growth factor - poly(lactic-co-glycolic-acid) microsphere.

(PBS, $\mathrm{pH}=7.4$ ) were added separately in every tube. Tubes 1a-24a, 1b-24b, and 1c-24c were placed in normal pressure, $0.35 \mathrm{MPa}$, and 4.0 MPa pressure loading equipment, respectively, while tubes $1 \mathrm{~d}-24 \mathrm{~d}$ were placed in thermostatic water bath oscillator $\left(37^{\circ} \mathrm{C}, 100 \mathrm{rpm}\right.$, normal pressure, amplitude: $30 \mathrm{~mm}$ ). The bFGF-PLGA MS present in supernatant fluid after centrifugation (three tubes of each group) was collected every week, respectively. The differences of bFGF-PLGA MS among those four groups, including microsphere morphology (observed by an FEI Quanta 200 scanning electron microscope; FEI, Hillsboro, OR, USA), microsphere quality, and average molecular weight of polymer (studied by a 150 CALC/GPC, Waters Corporation, Milford, MA, USA) were observed. 2) Approximately $20 \mathrm{mg}$ bFGF-PLGA MSs and $20 \mathrm{~mL}$ PBS were mixed and added into centrifugal tubes (three tubes of each group). The concentrations of bFGF in the supernatant fluid from the centrifuge tubes were measured at $0.5,1,3,6,9,12,15,18,21,24,27,30,33$ days, respectively. A sample of $1 \mathrm{~mL}$ was taken every time, and an equal amount of fresh PBS was added after sampling. By adopting the enzyme-linked immunosorbent assay technique, the absorbance of bFGF standard substance and sample solution were determined, and the releasing curves of the bFGF-PLGA MSs were drawn according to the accumulated release rate and time data.

The steps involved in the shearing force loading experiment are shown in Figure 3. A filter membrane (Shanghai,
People's Republic of China) with a $0.45 \mu \mathrm{m}$ aperture and $1 \mathrm{~kg} / \mathrm{cm}^{2}$ compression strength was placed on a filter board (with $10 \mathrm{~cm}$ diameter) in the middle of our self-made shearing force loading equipment (1a of Figure 3 ). The power system employed a Masterflex pulsating pump (Number 7550-17, Cole-Parmer, Vernon Hills, IL, USA) to provide stable flow field. A stainless steel micropore filter (Shanghai, People's Republic of China), which was installed between the filtration unit and pump, collected bFGF-PLGA MSs debris in the flow field. This micropore filter was changed every 3 days. The experiment took place at a constant temperature of $37^{\circ} \mathrm{C}$ and under normal pressure. The output of the experimental pulsating pump was $1,000 \mathrm{~mL} / \mathrm{min}$, while that of the control group was $10 \mathrm{~mL} / \mathrm{min}$. Approximately $150 \mathrm{mg}$ of bFGF-PLGA MSs and PBS were added into the equipment to set up an enclosed flow field system. Every week, 1/10 microspheres were removed after centrifugation and were freeze-dried for conservation and stored for 7 weeks. The drug release experiment was the same as that of static pressure loading experiment. The properties of bFGF-PLGA MSs including microsphere morphology, microsphere quality, weight-average molecular weight of polymer, and drug release were studied.

\section{Statistical analysis}

SAS 9.2 (SAS Institute Inc., Cary, NC, USA) software was used for statistical analysis. The values are presented as mean \pm standard deviation. Two-factor analysis of variance 


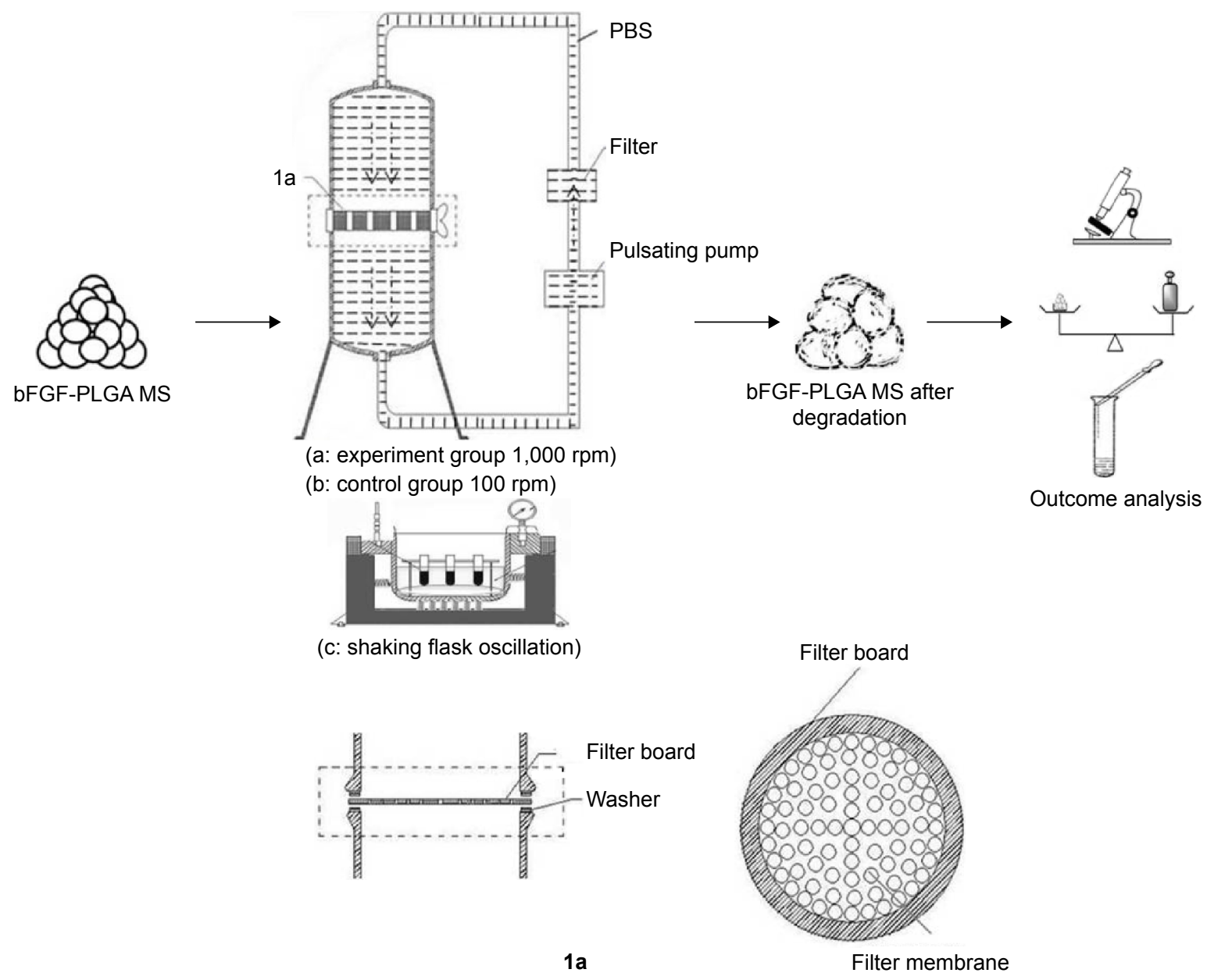

Figure 3 The shearing force load experiment equipment and experimental process of bFGF-PLGA MS degradation and drug release. Notes: I closeup details the vertical section of the filter board on the left and the cross section of the filter board on the right.

Abbreviations: bFGF-PLGA MS, basic fibroblast growth factor - poly(lactic-co-glycolic-acid) microsphere; PBS, phosphate-buffered saline.

(ANOVA) was used to determine the differences between two groups and multiple groups of mean values. Meanwhile, the interaction and effect of the two factors (stress and time) on bFGF-PLGA MS degradation and bFGF release were studied. $P \leq 0.05$ was considered significant.

\section{Results}

\section{The scanning electron microscope images of bFGF-PLGA MS}

In static pressure loading experiment, no obvious deformation and structure damage of microsphere was found initially. The microsphere morphology in shaking flask oscillator group changed greatly (Figure 4). After 2 weeks of degradation, the surface of the microsphere appeared as pits and tiny holes. Five weeks later, the holes increased in size and the microsphere dissolved gradually. After 8 weeks, the microsphere collapsed. The changes in the microsphere morphology in other groups were similar to that in the shaking flask oscillation group, just that there was a lesser extent of degradation.
In the 4.0 $\mathrm{MPa}$ pressure loading group, few holes began to appear after 5 weeks degradation in microspheres. Eight weeks later, the holes increased in size and the microspheres began to deform (Figure 5). In the $0.35 \mathrm{MPa}$ and normal pressure loading groups, the microsphere remained intact and pits and tiny holes appeared only after 8 weeks.

In shearing force loading experiment, the changes in microsphere morphology were similar in both the experimental and the control group. After 3 weeks of degradation in experimental group, the surface of microspheres became irregular, like golf balls. After 5 weeks, there were some tiny holes on the surface. Microspheres began to distort and adhere together. Seven weeks later, the holes increased in number and became larger, and some had perforation (Figure 6).

\section{The changes in bFGF-PLGA MS molecular weight}

In the static pressure loading experiment, the molecular weight of bFGF-PLGA MS during degradation constantly 


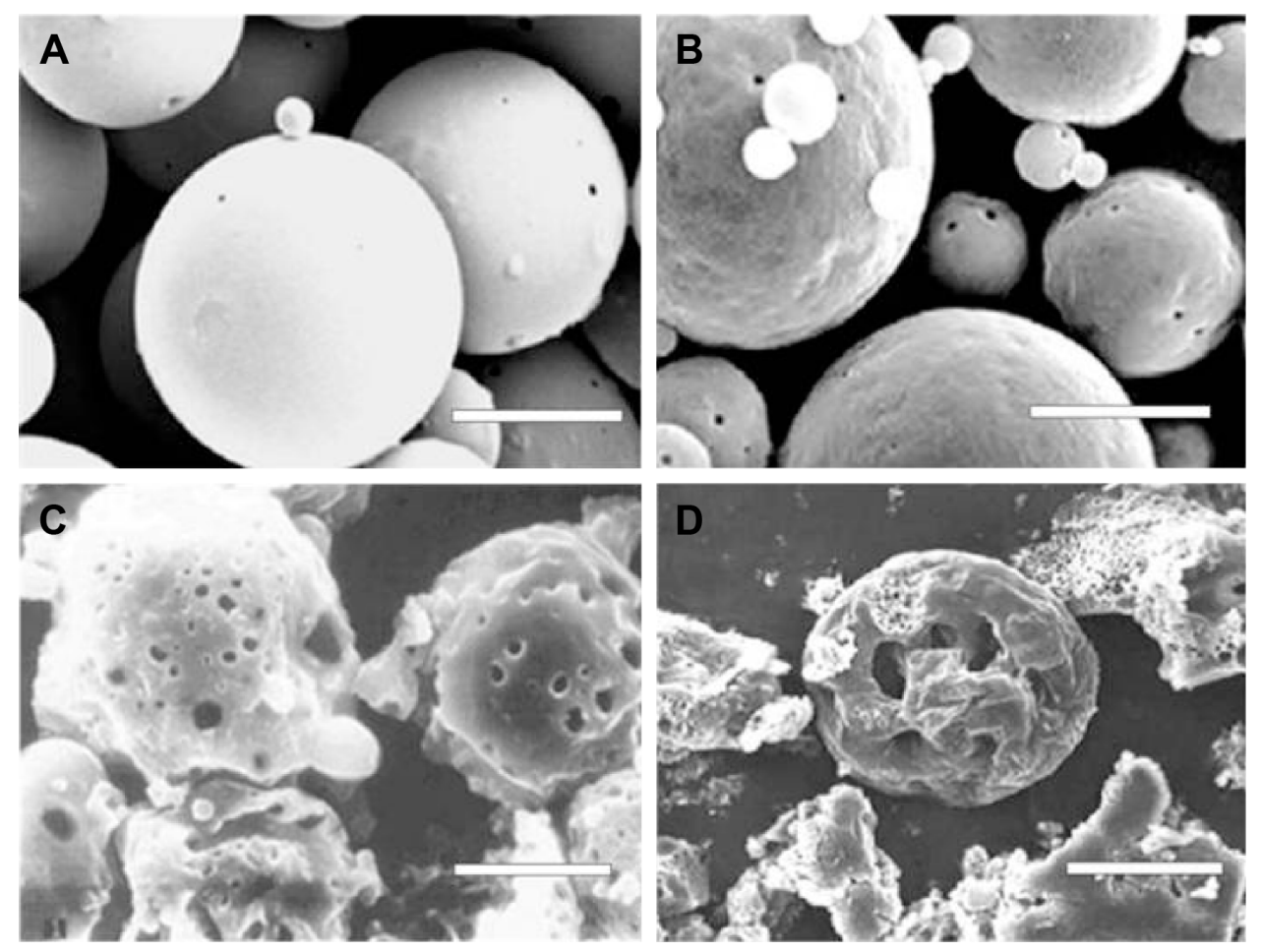

Figure 4 The scanning electron microscope images of bFGF-PLGA MS in shaking flask oscillation group.

Notes: (A) Before degradation. B-D are 2, 5, and 8 weeks after degradation, respectively. Scale bars, $5 \mu \mathrm{m}$ for (A-D).

Abbreviation: bFGF-PLGA MS, basic fibroblast growth factor - poly(lactic-co-glycolic-acid) microsphere.

reduced. The results are shown in Figure 7. The molecular weight of bFGF-PLGA MS in shaking flask oscillation group declined fastest of all, and fell by half after 4 weeks. After 8 weeks degradation, the molecular weight had fallen to $27 \%$, which was a statistically significant difference compared to the other three groups (ANOVA, $P<0.0001$ ). Meanwhile, the trend of the molecular weight in normal and $0.35 \mathrm{MPa}$ pressure loading groups were consistent (ANOVA, $P=0.9478$ ), and their molecular weight had dropped finally to $36 \%$. The molecular weight in 4.0 MPa pressure loading group declined in shaking flask oscillation group, and this was statistically significant compared to the normal pressure loading groups (ANOVA, $P<0.0001$ ). After 8 weeks degradation, the molecular weight had fallen to $51 \%$.

In shearing force loading experiment, shown in Figure 8, there was no significant difference between the experimental
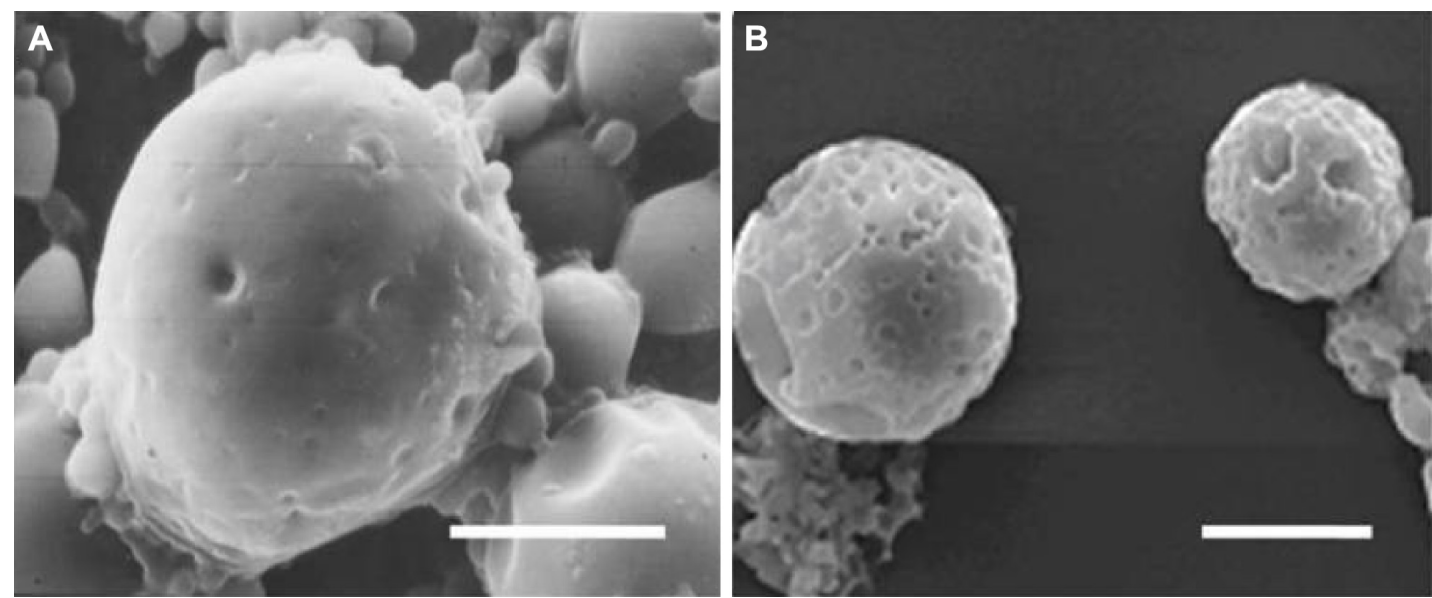

Figure 5 The scanning electron microscope images of bFGF-PLGA MS in 4.0 pressure loading group.

Notes: (A, B) 5, 8 weeks after degradation. Scale bars, $5 \mu \mathrm{m}$ for (A), $10 \mu \mathrm{m}$ for (B).

Abbreviation: bFGF-PLGA MS, basic fibroblast growth factor - poly(lactic-co-glycolic-acid) microsphere. 

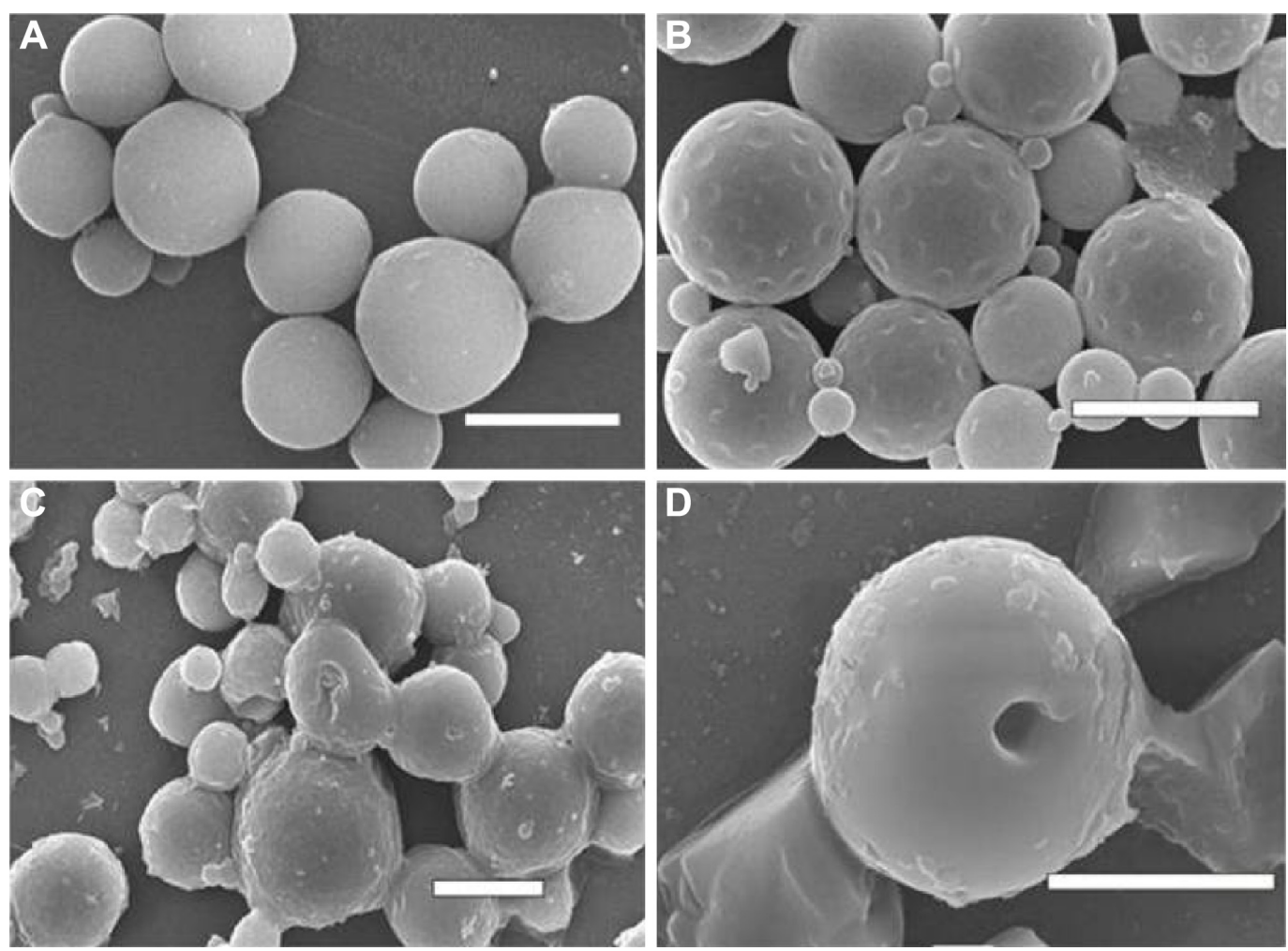

Figure 6 The scanning electron microscope images of bFGF-PLGA MS in shearing force loading experiment group.

Notes: (A) Before degradation, (B) 3 weeks, (C) 5 weeks, and (D) 7 weeks after degradation. Scale bars $5 \mu \mathrm{m}$ for (A, D), $10 \mu \mathrm{m}$ for (B, C).

Abbreviation: bFGF-PLGA MS, basic fibroblast growth factor - poly(lactic-co-glycolic-acid) microsphere.

and the control group (ANOVA, $P=0.3747$ ). After 49 days degradation, the molecular weight had dropped to $63 \%$ and $65 \%$, respectively. However, the degradation rate of molecular weight in shaking flask oscillation was much faster than that in shearing force loading experiment only (ANOVA, $P<0.0001$ ).

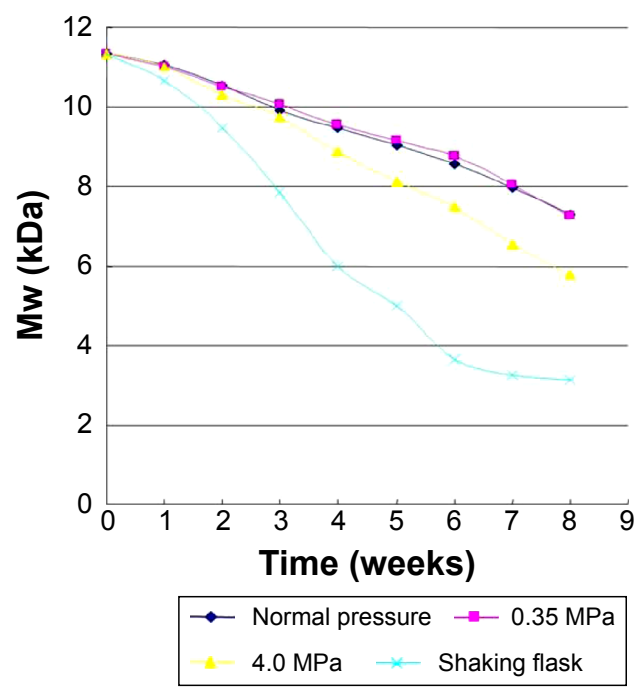

Figure 7 The molecular weight changes of bFGF-PLGA MS during degradation in the static pressure loading experiment.

Note: The average of initial Mw was II.32 $\pm 0.11 \mathrm{kDa}$.

Abbreviations: bFGF-PLGA MS, basic fibroblast growth factor - poly(lactic-coglycolic-acid) microsphere; Mw, molecular weight.

\section{Mass loss of bFGF-PLGA MS during degradation}

In the static pressure loading experiment, due to degradation, the mass of microspheres were getting reduced and the mass loss rate increased (Figure 9). After 8 weeks, the

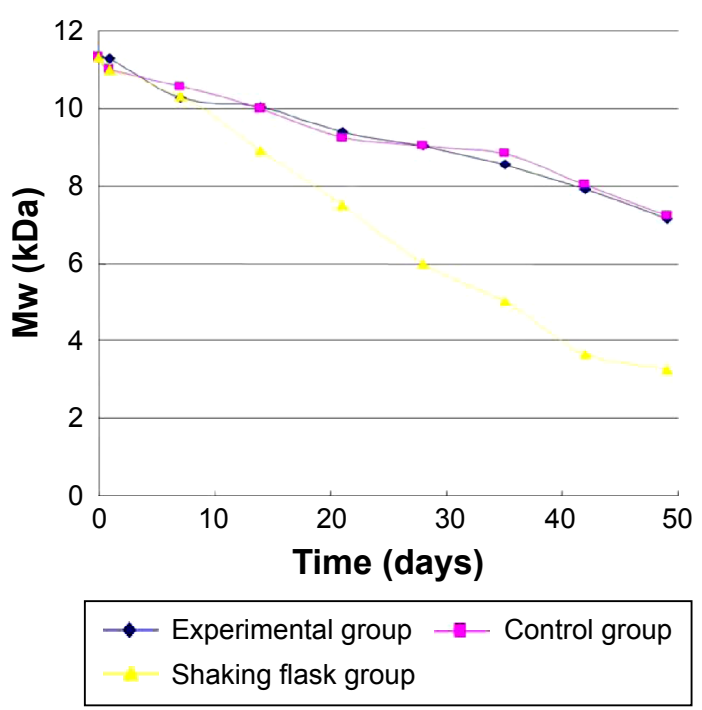

Figure 8 The molecular weight changes of bFGF-PLGA MS during degradation in shearing force loading experiment.

Abbreviations: bFGF-PLGA MS, basic fibroblast growth factor - poly(lactic-coglycolic-acid) microsphere; $\mathrm{Mw}$, molecular weight. 


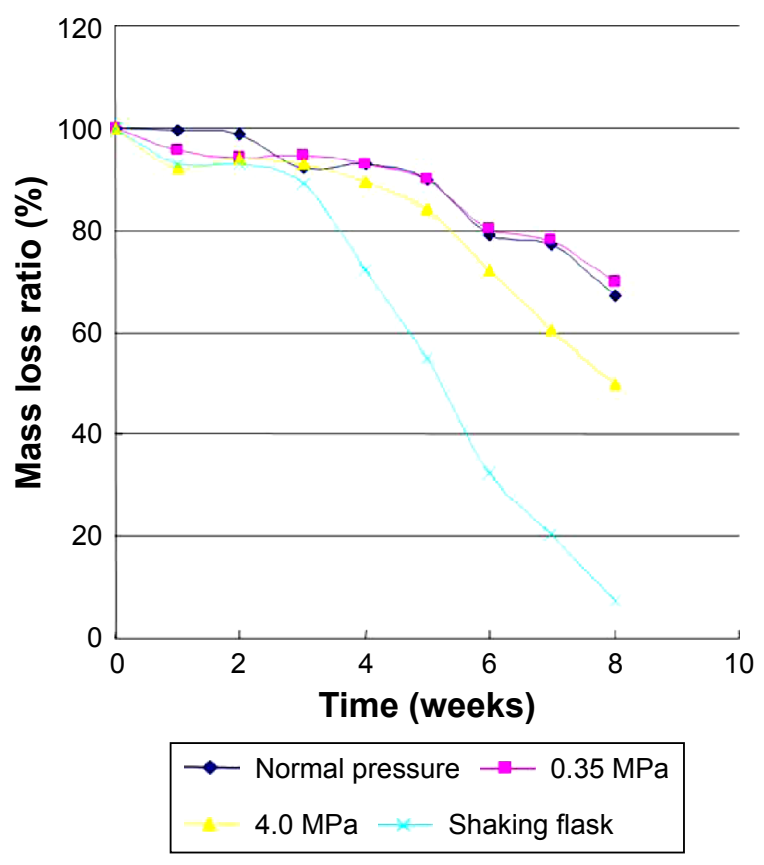

Figure 9 Mass loss of bFGF-PLGA MS during degradation in the static pressure loading experiment.

Abbreviation: bFGF-PLGA MS, basic fibroblast growth factor - poly(lactic-coglycolic-acid) microsphere.

mass loss ratio of microsphere had dropped $50 \%$ in the 4.0 MPa pressure loading group, which was higher than in the $0.35 \mathrm{MPa}(30 \%)$ and atmospheric pressure loading group (33\%) (ANOVA, $P=0.0001,0.0007)$. In shaking flask oscillation group, the mass loss ratio of microspheres was more obvious, especially 3 weeks later. Meanwhile, the mass loss ratio of microspheres in shaking flask oscillation group had fallen to $7 \%$ finally, which was statistically significant compared to other groups (ANOVA, $P<0.0001$ ).

In shearing force loading experiment, the mass of microspheres, both in the experimental and control groups, were reducing and were getting lost faster after 35 days. After 49 days, the mass loss ratio of microsphere had dropped to $58 \%$ and $65 \%$, respectively. There was no significant difference in mass loss between two groups (ANOVA, $P=0.0541$ ) (Figure 10). Meanwhile, the mass of microspheres in shaking flask group reduced the most compared with the other three groups finally (ANOVA, $P<0.0001$ ).

\section{Outcomes of drug releasing of bFGF- PLGA MS}

In static pressure loading experiment, shown as in Figure 11, there were two typical release phases of drug release. The drug releasing rate of each group was approximately $25 \%$, and there was little difference in initial release stage. In the second period, the drug releasing rate in shaking flask oscillation group obviously accelerated and had significant
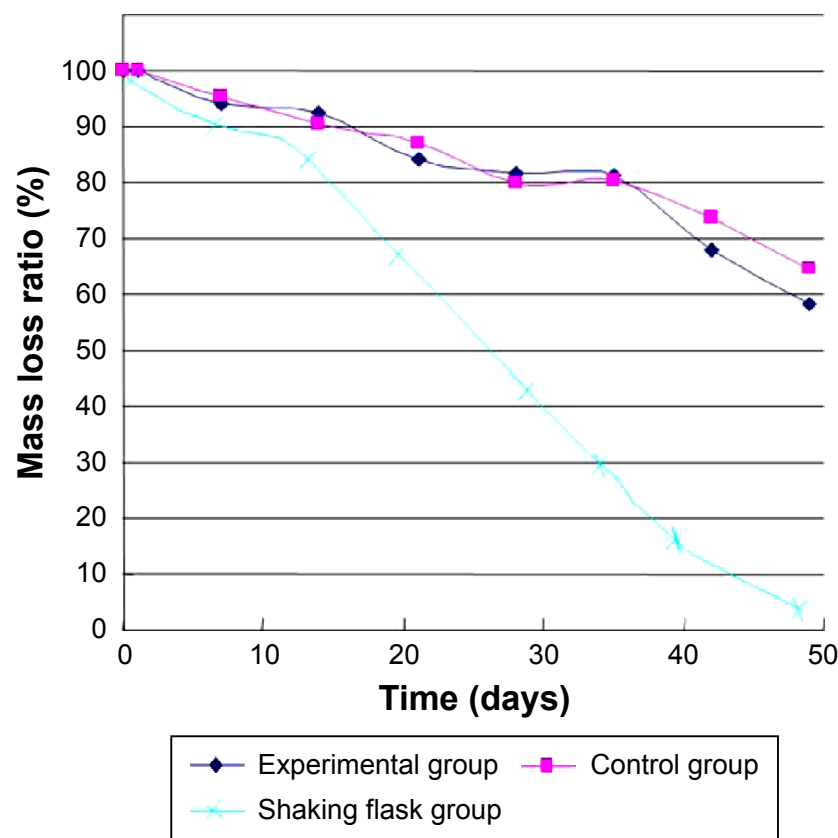

Figure 10 Mass loss of bFGF-PLGA MS during degradation in shearing force loading experiment.

Abbreviation: bFGF-PLGA MS, basic fibroblast growth factor - poly(lactic-coglycolic-acid) microsphere.

differences with other three groups (ANOVA, $P<0.001$ ). The rate rose to $80 \%$ in 21 days. The drug releasing rate of the 4.0 MPa group was faster than that of the $0.35 \mathrm{MPa}$ and normal pressure loading group. Meanwhile, there was no difference between the normal pressure and $0.35 \mathrm{MPa}$ group (ANOVA,
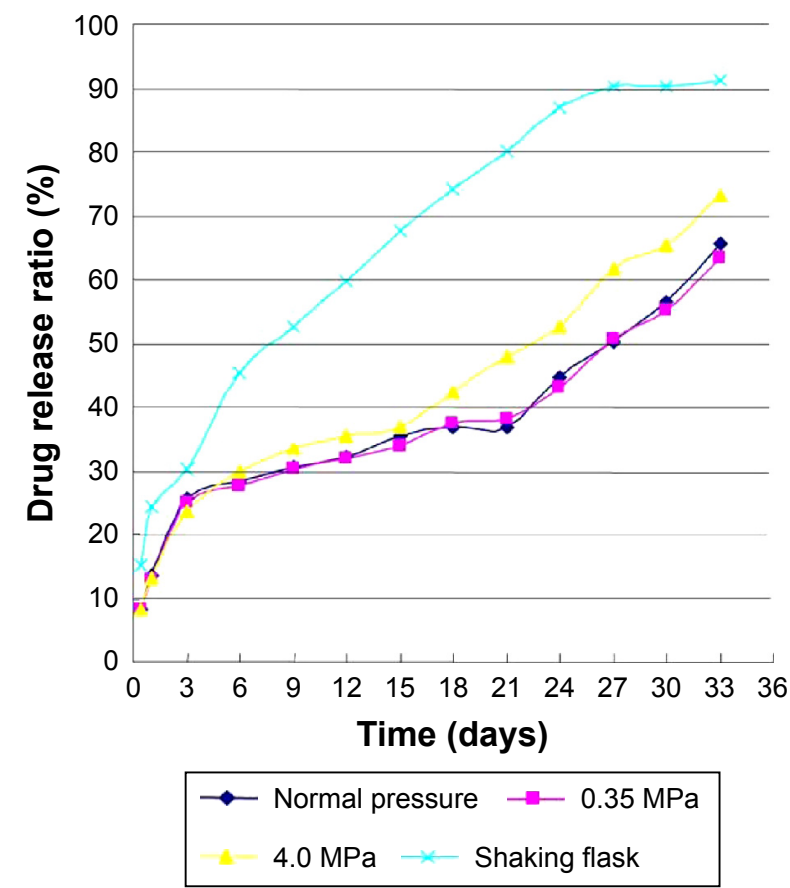

Figure I I Drug release of bFGF-PLGA MS during degradation in the static pressure loading experiment.

Abbreviation: bFGF-PLGA MS, basic fibroblast growth factor - poly(lactic-coglycolic-acid) microsphere. 


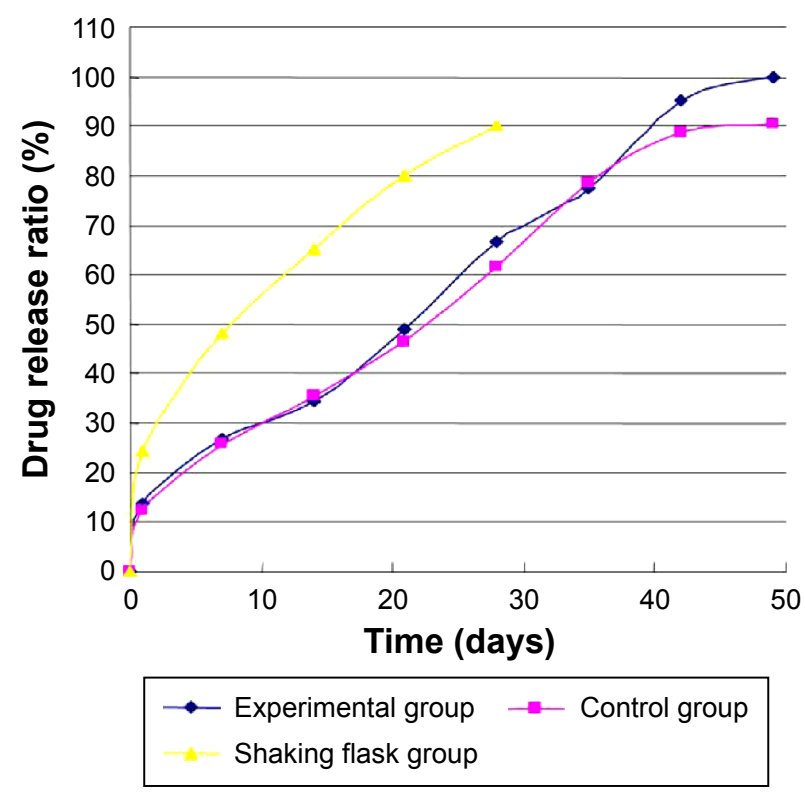

Figure 12 Drug release of bFGF-PLGA MS during degradation in shearing force loading experiment.

Abbreviation: bFGF-PLGA MS, basic fibroblast growth factor - poly(lactic-coglycolic-acid) microsphere.

$P=0.6256$ ). In shearing force loading experiment (Figure 12), there was no significant difference between experimental and control group (ANOVA, $P=0.0900$ ). However, the drug release rate in flask-shaking is much faster than in a single shear force (ANOVA, $P<0.0001$ ).

\section{Discussion}

Articular cartilage is frequently injured by disease, sportsrelated trauma, and so on. However, its repairing capacity is limited because of its avascular characteristics. ${ }^{11,12}$ In previous studies, microspheres embedding bFGF could effectively promote cartilage regeneration and repair in large chondral defects. ${ }^{12-14}$ To our knowledge, the complex environment of articular cavity, especially joint motion, impacted the bFGFPLGA MS degradation and drug release thereby affecting cartilage regeneration and repair.

There are two kinds of distribution when bFGF-PLGA MSs are injected into synovial fluid. The bFGF-PLGA MSs either can diffuse in the joint synovial fluid or adhere to the surface of synovium and joint cartilage. As joints are kept stationary, the bFGF-PLGA MSs bear static pressure. When the joint moves, the bFGF-PLGA MSs adhere to the surface and are mainly affected by shearing force, besides joint static pressure. However, stress of bFGF-PLGA MSs suspended in synovial fluid is complicated. The bFGF-PLGA MSs collide with each other, joint capsule, and cartilage, just like shaking flask oscillation.
In our study of static pressure loading experiment, the results showed that the bFGF-PLGA MSs in 0.35 and 4.0 MPa pressure groups had no obvious deformation and structure damage. It can be seen that the bFGF-PLGA MSs in joint cavity were stable. Although different load in joint cavity cannot affect bFGF-PLGA MS's stability, its degradation rate can be affected. Our study showed that the bFGFPLGA MS degradation rate at 4.0 MPa pressure accelerated. Particularly in the second phase, the molecular weight and the quality declined more quickly significantly than in the normal pressure and $0.35 \mathrm{MPa}$ groups. The reasons may be as follows: ${ }^{25-28}$ first of all, deformation of bFGF-PLGA MS by load produces strain energy, which promotes molecular chain movement. Second, the load makes the molecules of PLGA rearrange and the molecular chain segments become motor, which converts into chemical energy and reduces hydrolysis energy barrier, thus increasing moisture absorption and degradation. In addition, the load can directly affect the mechanical properties of materials, such as reducing the modulus of elasticity and increasing brittleness, which cause the branched chain breaking.

In shearing force loading experiment, there was no significant difference between experimental and control group. Therefore, it can be thought that there was a slight effect of the shearing force on the degradation of microspheres. When the joint moved, the surface of joint cartilage normally did not contact closely and there was a thin layer of synovial fluid existing between the surfaces of two cartilages. It is regarded that the shearing force had a slight effect on the degradation of microspheres in joint cavity, because of the relatively low shearing force in synovial fluid. The degradation rate of microspheres in shaking flask oscillation was higher than that only in shearing force. Some factors that may affect degradation rate are as follows: the first is that microspheres can diffuse evenly and contact sufficiently in the synovial fluid during shaking flask oscillation. Thus, the area of hydrolysis increases. The other is that deformation of microsphere is caused during shaking flask oscillation. Some hollows could be observed on the surface of microspheres by the scanning electron microscope, 2 weeks later. By this time, microspheres looked like golf balls (Figure 4). Meanwhile, kinetic energy and thermal energy are developed, which convert into chemical energy to accelerate microsphere degradation.

The drug release of microspheres can be divided into two stages: a sudden release phase (burst release phase) and steady release phase..$^{29-33}$ As we know, the kinetics and mechanism of peptide drugs cause free direct release 
of protein molecules, which adsorb on the surface of the microspheres, causes sudden release phase. Stable release phase is the main carrier drug microsphere drug release phase, which is controlled by the diffusion and degradation processes. Thus, drugs can take a long time to maintain a steady drug release rate. In steady release phase, hydrated polymer degradation, polypeptide, and protein molecules diffusing through the microporous structure of the microspheres by hydrolyzation form the first-line release phase. Finally, quality loss and polymer protein releasing along the PLGA erosion process compose the second linear phase. This study showed that load rarely affected the drug release rate in the static pressure loading experiment. The drug release rate of the 4.0 MPa group was quicker than the atmospheric and $0.35 \mathrm{MPa}$ pressure group. Maybe the reason is that $4.0 \mathrm{MPa}$ pressure speeded up degradation rate of PLGA microspheres, especially in steady release phase. In shearing force loading experiments, the drug releasing rate of microsphere was relatively high. However, there was no significant difference of the drug releasing rates between the experimental and control group. It can be regarded that there was no effect of shearing force in flow field on the drug dissolution. The drug releasing rate of shaking flask oscillation was far higher than those of the two groups, and this was related to acceleration of microsphere degradation rate. It is indicated that shaking flask oscillation had an effect on the drug releasing of microspheres.

\section{Conclusion}

The bFGF-PLGA MS in joint cavity are stable. The lower load has slight effect on microsphere degradation and drug release, but the higher load (4.0 MPa) has more. The shearing force in flow field under the current condition has slight effect on microsphere degradation and drug release. Microsphere degradation and drug release during shaking flask oscillation are faster than those of shearing force only.

\section{Acknowledgment}

This study was supported by the National Natural Science Foundation of China (10402025).

\section{Disclosure}

The authors report no conflicts of interest in this work.

\section{References}

1. Okada-Ban M, Thiery JP, Jouanneau J. Fibroblast growth factor-2. Int J Biochem Cell Biol. 2000;32(3):263-267.

2. Ornitz DM, Itoh N. Fibroblast growth factors. Genome Biol. 2001; 2(3):1-12.
3. Levenstein ME, Ludwig TE, Xu RH, Llanas RA. Basic fibroblast growth factor support of human embryonic stem cell self-renewal. Stem Cells. 2006;24(3):568-574.

4. Shen B, Pei F, Duan H, et al. Preparation and in vitro activity of controlled release microspheres incorporating bFGF. Chin J Traumatol. 2008;11(1):22-27.

5. Fei Y, Hurley MM. Role of fibroblast growth factor 2 and Wnt signaling in anabolic effects of parathyroid hormone on bone formation. $J$ Cell Physiol. 2012;227(11):3539-3545.

6. Grothe C, Nikkhah G. The role of basic fibroblast growth factor in peripheral nerve regeneration. Anat Embryol. 2001;204(3): 171-177.

7. Turner N, Grose R. Fibroblast growth factor signalling: from development to cancer. Nat Rev Cancer. 2010;10(2):116-129.

8. Anderson JM, Shive MS. Biodegradation and biocompatibility of PLA and PLGA microspheres. Adv Drug Deliv Rev. 2012;64:72-82.

9. Hickey T, Kreutzer D, Burgess DJ, Moussy F. Dexamethasone/ PLGA microspheres for continuous delivery of an anti-inflammatory drug for implantable medical devices. Biomaterials. 2002;23(7): $1649-1656$.

10. Ford Versypt AN, Pack DW, Braatz RD. Mathematical modeling of drug delivery from autocatalytically degradable PLGA microspheres a review. J Control Release. 2013;165(1):29-37.

11. Hunziker EB. Articular cartilage repair: basic science and clinical progress. A review of the current status and prospects. Osteoarthritis Cartilage. 2002;10(6):432-463.

12. Buckwalter JA. Articular cartilage injuries. Clin Orthop Relat Res. 2002;402:21-37.

13. Fujisato T, Sajiki T, Liu Q, Ikada Y. Effect of basic fibroblast growth factor on cartilage regeneration in chondrocyte-seeded collagen sponge scaffold. Biomaterials. 1996;17(2):155-162.

14. Fortier LA, Barker JU, Strauss EJ, McCarrel TM, Cole BJ. The role of growth factors in cartilage repair. Clin Orthop Relat Res. 2011; 469(10):2706-2715.

15. Fukuda A, Kato K, Hasegawa M, et al. Enhanced repair of large osteochondral defects using a combination of artificial cartilage and basic fibroblast growth factor. Biomaterials. 2005;26(20):4301-4308.

16. Zou GK, Song YL, Zhou W, et al. Effects of local delivery of bFGF from PLGA microspheres on osseointegration around implants in diabetic rats. Oral Surg Oral Med Oral Pathol Oral Radiol. 2012; 114(3):284-289.

17. Reeve MS, McCarthy SP, Downey MJ, Gross R. Polylactide stereochemistry: effect on enzymatic degradability. Macromolecules. 1994;27(3):825-831.

18. Grayson ACR, Cima MJ, Langer R. Size and temperature effects on poly(lactic-co-glycolic acid) degradation and microreservoir device performance. Biomaterials. 2005;26(14):2137-2145.

19. Fu K, Pack DW, Klibanov AM, Langer R. Visual evidence of acidic environment within degrading poly (lactic-co-glycolic acid)(PLGA) microspheres. Pharm Res. 2000;17(1):100-106.

20. Stromqvist B, Nilsson LT, Egund N, Thorngren KG, Wingstrand HA. Intracapsular pressures in undisplaced fractures of the femoral neck. J Bone Joint Surg Br. 1988;70(2):192-194.

21. Flanigan DC, Lin F, Koh JL, Zhang LQ. Articular contact pressures of meniscal repair techniques at various knee flexion angles. Orthopedics. 2010;33(7):475-485.

22. Guettler JH, Demetropoulos CK, Yang KH, Jurist KA. Dynamic evaluation of contact pressure and the effects of graft harvest with subsequent lateral release at osteochondral donor sites in the knee. Arthroscopy. 2005;21(6):715-720.

23. Bos RRM, Rozema FR, Boering G, Nijenhuis AJ, Pennings AJ, Verwey AB. Bio-absorbable plates and screws for internal fixation of mandibular fractures. A study in six dogs. Int J Oral Maxillofac Surg. 1989;18(6):365-369.

24. Korin N, Kanapathipillai M, Matthews BD, et al. Shear-activated nanotherapeutics for drug targeting to obstructed blood vessels. Science. 2012;337(6095):738-742. 
25. Schliecker G, Schmidt C, Fuchs S, Wombacher R, Kissel T. Hydrolytic degradation of poly(lactide-co-glycolide) films: effect of oligomers on degradation rate and crystallinity. Int J Pharm. 2003;266(1-2):39-49.

26. Farrar DF, Gillson RK. Hydrolytic degradation of polyglyconate B: the relationship between degradation time, strength and molecular weight. Biomaterials. 2002;23(18):3905-3912.

27. Göpferich A. Mechanisms of polymer degradation and erosion. Biomaterials. 1996;17(2):103-114.

28. Yang Y, Tang G, Zhao Y, Yuan X, Fan Y. Effect of cyclic loading on in vitro degradation of poly(L-lactide-co-glycolide) scaffolds. J Biomater Sci Polym Ed. 2010;21(1):53-66.

29. Lu L, Yaszemski MJ, Mikos AG. TGF- $\beta 1$ release from biodegradable polymer microparticles: its effects on marrow stromal osteoblast function. J Bone Joint Surg Am. 2001;83-A(Suppl 1 Pt2):S82-S92.
30. Messaritaki A, Black SJ, van der Walle CF, Rigby S. NMR and confocal microscopy studies of the mechanisms of burst drug release from PLGA microspheres. J Control Release. 2005;108(2):271-281.

31. Ford Versypt AN, Pack DW, Braatz RD. Mathematical modeling of drug delivery from autocatalytically degradable PLGA microspheres - a review. J Control Release. 2013;165(1):29-37.

32. Fredenberg S, Wahlgren M, Reslow M, Axelsson A. The mechanisms of drug release in poly (lactic-co-glycolic acid)-based drug delivery systems - a review. Int J Pharm. 2011;415(1):34-52.

33. Makadia HK, Siegel SJ. Poly lactic-co-glycolic acid (PLGA) as biodegradable controlled drug delivery carrier. Polymers. 2011;3(3): $1377-1397$.

\section{Publish your work in this journal}

Drug Design, Development and Therapy is an international, peerreviewed open-access journal that spans the spectrum of drug design and development through to clinical applications. Clinical outcomes, patient safety, and programs for the development and effective, safe, and sustained use of medicines are a feature of the journal, which has also been accepted for indexing on PubMed Central. The manuscript management system is completely online and includes a very quick and fair peer-review system, which is all easy to use. Visit http://www.dovepress.com/testimonials.php to read real quotes from published authors.

Submit your manuscript here: http://www.dovepress.com/drug-design-development-and-therapy-journal 